\title{
THERMOSPHERE AND IONOSPHERE DYNAMICS DURING 20-30 MARCH 1979 TIME PERIOD: COMPARISON OF TIGCM CALCULATED DENSITIES WITH OBSERVATIONS
}

\author{
F. A. Marcos, * R. G. Roble** and T. L. Killeen*** \\ * Air Force Geophysics Laboratory, Hanscom AFB, MA 01731, U.S.A. \\ ** High Altitude Observatory, National Center for Atmospheric Research, \\ Boulder, CO 80307, U.S.A. \\ *** Space Physics Research Laboratory, University of Michigan, Ann Arbor, \\ MI 48109, U.S.A.
}

\author{
ABSTRACT
}

The Thermosphere Ionosphere General Circulation Model developed at the National Center for Atmospheric Research, (NCAR TIGCM), has been used to simulate the time-dependent variations of global thermosphere and ionosphere structure and dynamics during the 20-30 March 1979 time period. Thermospheric density variations predicted by the TIGCM during this period are statistically compared to satellite electrostatic triaxial accelerometer neutral density measurements obtained between 170 and $240 \mathrm{~km}$ altitude and to predictions made by the Mass Spectrometer Incoherent Scatter empirical model (MSIS-86). In its present state of development, the TIGCM has attained about the same accuracy (standard deviation), as MSIS-86. Incorporation of improved representation of ion drag, resulting from the downward flow of magnetospheric plasma on the nightside, has contributed to the TIGCM model accuracy.

\section{INTRODUCTION}

Validation of complex numerical models such as the NCAR TIGCM / $1 /$ and the University College of London model $/ 2 /$ is required for assessing their applicability for geophysical studies and for practical applications such as satellite drag prediction. Quantitative model assessments have been limited due to a lack of accurate measurements of basic parameters in the thermosphere. Extensive measurements of one parameter, neutral density, are available from the Satellite Electrostatic Triaxial Accelerometer (SETA) $/ 3 /$. This instrument measures satellite drag forces which are directly proportional to density. Northern hemisphere data obtained in a sun-synchronous $1000 / 2200 \mathrm{hr}$ local time orbit are used in this analysis. The data for each pass typically starts at 240 $\mathrm{km}$ altitude on the nightside equator, reaches about $200 \mathrm{~km}$ near the pole, is at minimum altitude of $170 \mathrm{~km}$ near 40 degrees north latitude on the dayside and rises to $180 \mathrm{~km}$ at the equator. Accelerometer data previously used to evaluate several empirical neutral density models showed that while our understanding of the morphology of atmospheric variations has increased significantly, there has been little progress in quantitatively representing the variability $/ 4 /$. The model errors tend to maximize at high latitudes where they are sensitive to geomagnetic activity. The present study provides the first statistical evaluation of the TIGCM capability to eventualiy improve on the neutral density accuracies achieved by current empirical models.

\section{TIGCM SIMULATIONS}

The TIGCM extends the NCAR thermospheric general circulation model (NCAR TGCM) by incorporating a self-consistent ionosphere. The TGCM is a three-dimensional, time-dependent model of the neutral upper atmosphere. The model uses a finitedifferencing technique to obtain self-consistent, time dependent solutions to the coupled, nonlinear equations of hydrodynamics, thermodynamics and continuity of the neutral gas and to the coupling between the dynamics and the composition /5-7/. The TIGCM development includes a self-consistent aeronomic scheme of the thermosphere and ionosphere. Nutual couplings between the thermospheric neutral gas and ionospheric plasma occur at each model time step and at each point of the geographic grid. Parameterizations of various input fields such as solar EUV and UV fluxes, auroral particle precipitation, ionospheric convection pattern, and the amplitudes and phases of semi-diurnal tides from the lower atmosphere are necessary for the simulation of the three-dimensional time-dependent JASR 12:6-G 


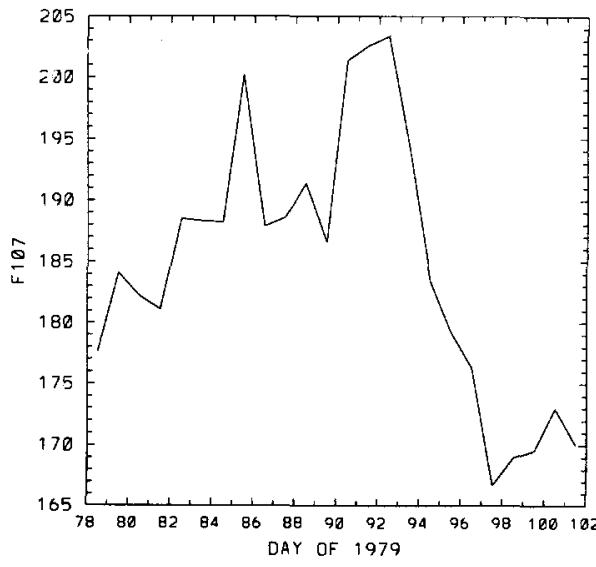

(a)

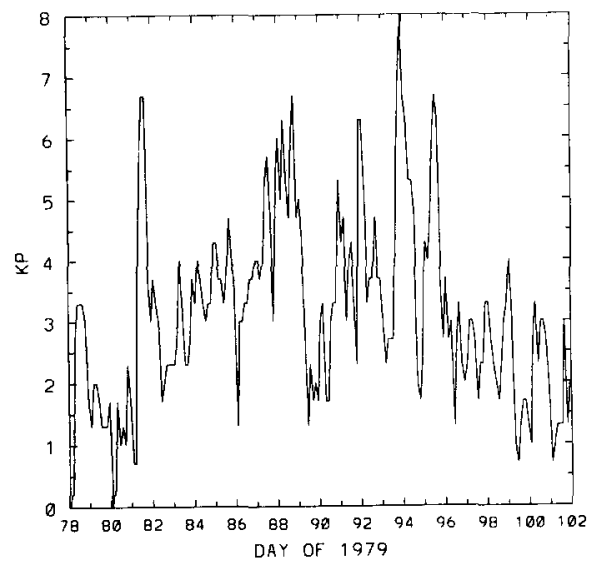

(b)

Fig. 1. (a) Daily $\mathrm{F}_{10,7} \mathrm{~cm}$ solar flux, and (b) 3-hr $\mathrm{kp}$ index including period of this study (days 79-89).

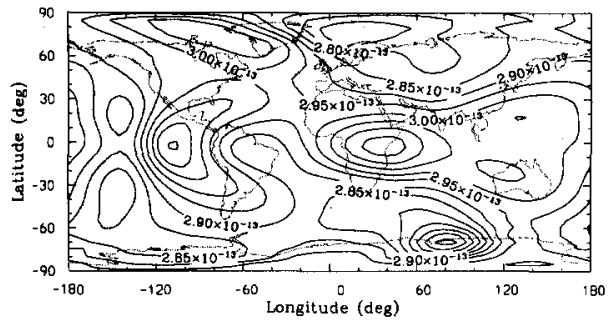

(a)

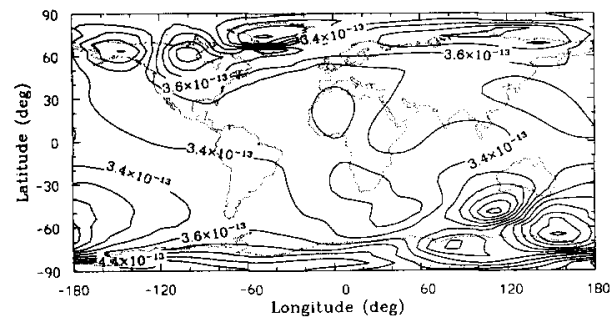

(b)

Fig. 2. TIGCM global simulations of total mass density ( $\left.9 \mathrm{~cm}^{-3}\right)$ at an altitude of $200 \mathrm{~km}$ corresponding to geomagnetic conditions of (a) $\mathrm{Kp}=1+$ and (b) $\mathrm{Kp}=6+$.

physical processes described in these models. The model output is a global data set describing the structure and dynamics of the neutral and ionized atmosphere. Figure 1 shows the solar and geomagnetic data for March 20-30, 1979 (days 7989). This time period is characterized by high solar flux and moderate geomagnetic activity. The version of the TIGCM described in /1/ had deficiencies in the nighttime $F$ layer at low and middle latitudes. Simulations in this paper use an updated version of the model with a more realistic nighttime ionosphere obtained by prescribing a downward flow of plasma from the magnetosphere. For comparison with the satellite accelerometer density measurements, TIGCM total density is calculated by appropriately summing $\mathrm{N}_{2}, \mathrm{O}_{2}$ and 0 number densities. Figure 2 shows examples of the TIGCM neutral density simulations at $200 \mathrm{~km}$ as a function of latitude and local time at a fixed universal time for both quiet $\left(\mathrm{Kp}={ }^{1+}+\right)$ and disturbed $(\mathrm{Kp}=6+)$ geomagnetic conditions. Densities (units of $10^{-13} \mathrm{~g} \mathrm{cm-3}$ ) are typically $2.9 \pm 1$ at all locations for quiet conditions. For disturbed conditions, the density varies significantly with latitude. At low latitudes density values are about 3.4. Peak values, occurring at high latitudes, are 4.4 in the Southern Hemisphere and 4.2 in the Northern Hemisphere (near 75 deg latitude, - 50 deg longitude).

\section{VALIDATION OF TIGCM}

Data-to-model comparisons were carried out as a function of latitude, local time and geomagnetic activity. The accelerometer data for the period 20-30 Mar 79 were binned as follows: (a) all northern hemisphere, day, (b) all northern hemisphere, night, (c) 30 degree latitude bins with day/night, partitioning, and (d) 30 degree bins with day/night and geomagnetic partitioning. Two geomagnetic bins, 0 to $2+$ and 4 to 9 , were analyzed. The number of data points is nominally 12,000 for (a) and (b), 4000 for (c), and 2000 for the low $\mathrm{Kp}$ bin and 700 for the high $\mathrm{Kp}$ bin in (d). 


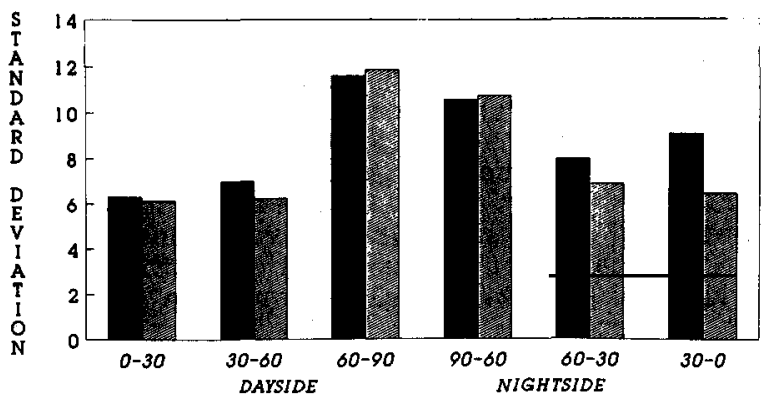

(a)

GEOGRAPHIC LATITUDE

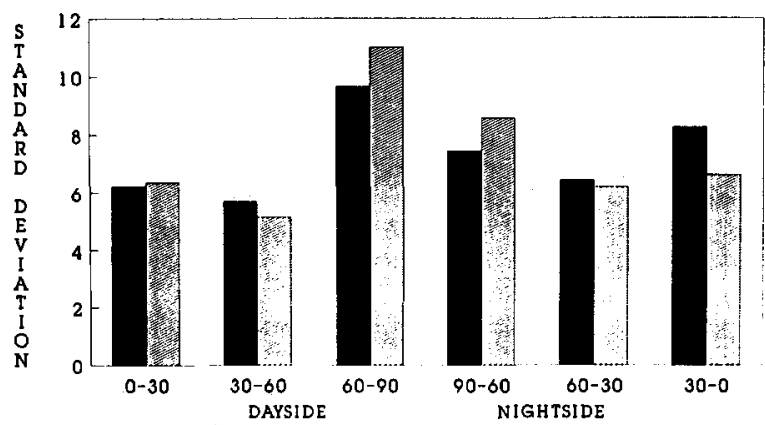

(b)

GEOGRAPHIC LATITUDE

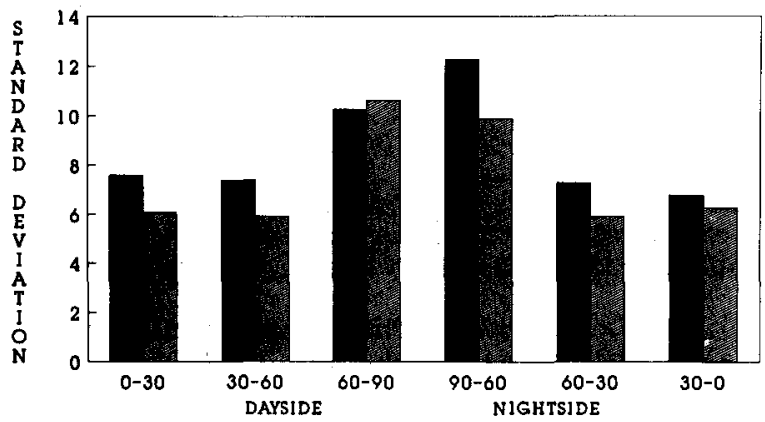

(c)

GEOGRAPHIC LATITUDE

TIGCM RTIS

Fig. 3. Percent standard deviation of satellite accelerometer measured neutral density to model predictions as a function of geographic latitude. The geographic pole is at the center; dayside data are to the left and nightside data are to the right. Results are for three cases: (a) data not binned according to geomagnetic conditions, (b) data in low geomagnetic activity bin, $\mathrm{Kp}$ range 0 to $2+$ and $(\mathrm{c})$ data in high geomagnetic activity bin, $\mathrm{Kp}$ range 4 to 9 .

The quantity statistically analyzed is the standard deviation of the ratios between corresponding measured and model densities. For each bin, standard deviation is computed by taking the difference, point by point between each ratio and the average ratio. For all nighttime data, with no binning, the standard deviation using TIGCM is $9.22 \%$, while for MSIS/8/ it is $8.41 \%$. On the dayside, standard deviations are $8.98 \%$ for TIGCM and $8.74 \%$ for MSIS. These results demonstrate that although the TIGCM has been tested and constrained mainly using data above $250 \mathrm{~km}$, its accuracy in depicting neutral density variability at altitudes below $250 \mathrm{~km}$ is now similar to that given by the 
empirical models. More detailed analyses of these statistical comparisons are given in Figure 3. Part (a) of Fig. 3 shows model errors for the 30 degree latitude and day/night binning without geomagnetic activity bins, while parts (b) and (c) show the data further partitioned, into low and high $\mathrm{kp}$ bins respectively. In these diagrams, the north geographic pole is at the center and the dayside and nightside equators are respectively to the left and to the right. The most striking aspect of all three cases is the similarity, rather than difference, between the standard deviations of the theoretical and empirical models. In all cases, the standard deviations are highest in the high latitude bins for both models. The model errors are generally within about $1.5 \%$ of each other. MSIS errors are about $2.5 \%$ less than those of TIGCM on the nightside at low latitudes and for high $\mathrm{kp}$ conditions at high latitudes. TIGCM errors axe lower than those of MSIS for dayside high latitude data and, except for high $\mathrm{Kp}$ conditions, for nightside high latitude conditions. While the data are not shown here, the effect of using an improved ionospheric boundary condition was to lower the TIGCM standard deviation by almost 58 in the nighttime mid- and low-latitude bins. This demonstrates the importance of ion drag on the temporal and spatial neutral density distribution.

\section{SUMMARY}

Physical models of the neutral atmosphere are now achieving the accuracy of empirical models. Thermospheric densities predicted by the TIGCM and MSIS models have been quantitatively assessed statistically by comparison to direct satellite accelerometer density measurements in the $170-240 \mathrm{~km}$ altitude region. TIGCM predictions of density variations at solar maximum, equinoctial conditions are comparable with the predictions made by MSIS. Specification of realistic ion drag fields in the TIGCM contributes to its accuracy. These results indicate that the major physical and chemical processes operating or affecting the region near $200 \mathrm{~km}$ have been identified. More accurate knowledge of thermospheric forcing functions which drive the model are required to improve forecasts of the neutral atmosphere.

\section{REFERENCES}

1. R.G. Roble, E. C. Ridley and A. D. Richmond, A coupled thermosphereionosphere general circulation model, Geophys. Res. Lett. 15, 1325 (1988).

2. T.J. Fuller-Rowell, D. Rees, S. Quegan, R.J. Moffett, and G. J. Bailey, Interactions between neutral thermospheric composition and the polar ionosphere using a coupled ionosphere-thermosphere model, J. Geophys Res. 92, 7744, (1987).

3. F.A. Marcos and J. M. Forbes, Thermospheric winds from the satellite electrostatic triaxial accelerometer system, J. Geophys. Res. 90, 6543 (1985).

4. F.A. Marcos, Accuracy of atmospheric drag models at low satellite altitudes, Adv. Space Res, 10, (3) 417 (1990).

5. R.E. Dickinson, E. C. Ridley and R.G. Roble, A three-dimensional, timedependent general circulation model of the thermosphere, J. Geophys. Res. 86, 1499 (1981).

6. R.G. Roble, R. E. Dickinson and E.C. Ridley, Global circulation and temperature structure of the thermosphere with high-latitude plasma convection, J. Geophys. Res, 87, 1599 (1982)

7. R.G. Roble and E. C. Ridley, An auroral model for the NCAR thermospheric general circulation model (TGCM), Ann. Geophys, 5A, 369 (1987).

8. A.E. Hedin, MSIS-86 thermospheric model, J. Geophys, Res. 92, 4649 (1987). 